\title{
Indigenous peoples and collaborative forest governance in northern forests: examining changes in policies, institutions, and communities - a selection of research papers presented at the ISSRM Conference, June 2017, Umea, Sweden, and the IUFRO Division 5 Conference, June 2017, Vancouver, Canada
}

\section{Special Editors}

Sara Teitelbaum, Département de sociologie, Université de Montréal, Pavillon Lionel Groux, C.P. 6124, succ Centre-Ville, Montréal, QC H3C 3J7, Canada

Stephen Wyatt, École de foresterie, Université de Moncton, Campus d’Edmundston, Pavillon de Foresterie, 165, Boul. Hébert, Edmunston, NB E3V 2S8, Canada

Ryan Bullock, Department of Environmental Studies and Sciences, The University of Winnipeg, 515 Portage Avenue, Winnipeg, MB R3B 2E9, Canada 